\title{
Joint Sub-Nyquist Spectrum Sensing Scheme with Geolocation Database over TV White Space
}

\author{
Yuan Ma, Student Member, IEEE, Xingjian Zhang, Student Member, IEEE, and Yue Gao, Senior Member, IEEE,
}

\begin{abstract}
To maximise spectrum access opportunities for white space devices, incorporating real-time spectrum sensing with geolocation database is a promising approach to enhance detection resolution with reduced computation complexity. Advanced spectrum sensing techniques are needed to quickly and accurately identify spectrum occupancy over a wide frequency range. However, the stringent requirements from wideband signal acquisition and processing pose a major implementation challenge in compact devices with limited energy storage and computation capabilities. In this paper, a hybrid scheme of subNyquist wideband spectrum sensing with geolocation database is proposed to achieve accurate detection of the surrounding spectrum with reduced number of required measurements and computation complexity. Two iterative algorithms are modified to incorporate a priori information from geolocation database, therefore enabling spectrum sensing to be performed only on a limited number of potentially vacant channels over TV White Space. Theoretical analyses and simulation results show that the proposed joint scheme speeds up the sensing process with enhanced detection performance and smaller required sampling rate, while the updated channel information from wideband spectrum sensing reduces the risk of interferences to the dynamic incumbent users.
\end{abstract}

Index Terms-Wideband Spectrum Sensing, Sub-Nyquist Rate Sampling, TV White Space, Simultaneous Orthogonal Matching Pursuit, $\ell_{v}$ norm minimisation.

\section{INTRODUCTION}

The rapid growth of Internet of things and mobile services is overwhelming current static spectrum supply, and thus encouraging an urgent need for improved and dynamic spectrum usage to mitigate the spectrum supply-demand gap [1]. There is an increased interest in the promising technique of spectrum sharing to facilitate efficient use of the spectrum driven by the following three factors: first, there is a significant spectrum crunch faced by the commercial mobile broadband users with the compelling need to get additional spectrum for the wireless broadband services [2]; second is the awareness that many licensed frequency bands are underutilised in practice either over time or geography [3]; finally, there have been some rapid advances towards the development of dynamic spectrum access through approaches such as geolocation database and cognitive radio [4].

The threat of spectrum scarcity has encouraged the governments to take critical steps towards releasing multiple bands for dynamic spectrum sharing, such as TV White Space (TVWS) [5], [6], Citizens Broadband Radio Service

Yuan Ma, Xingjian Zhang, and Yue Gao are with the School of Electronic Engineering and Computer Science, Queen Mary University of London, London E1 4NS, U.K. (emails:\{y.ma, xingjian.zhang, yue.gao\}@qmul.ac.uk).
(CBRS) in the $3.5 \mathrm{GHz}$ band [7], and the $5 \mathrm{GHz}$ unlicensed bands [8]. TVWS is the first major instance of spectrum sharing to be considered, which refers to the unused TV channels among the active ones in the ultra-high frequency (UHF) spectrum [9]. The experiences and concepts of utilising TVWS can also be extended to other white space bands. Compared with traditional Wi-Fi, the superior penetration propagation characteristic over UHF spectrum enables TVWS to have longer communication distance and better penetration through obstacles [10]. It has since been shown that over 50\% of locations in the UK are likely to have more than $150 \mathrm{MHz}$ of unutilized TV spectrum and that even $90 \%$ of locations might have around $100 \mathrm{MHz}$ of spectrum available [11]. Hence, making this spectrum available for reuse could bring substantial value to the citizens and consumers [12]. The UK communication regulator, Office of Communications (Ofcom), has announced the licence exempt regulations for TVWS in December 2015 [5]. Compact and low-power white space devices (WSDs) for rural broadband/WiFi-like accesses could therefore operate on the vacant channels [13].

To enable dynamic spectrum access over white space, fast and accurate detection of the spectrum is crucial to ensure that there is no harmful interference caused to the surrounding licensed services, including Digital Terrestrial Televisions (DTT), Programme Making and Special Events (PMSE) users, e.g., wireless microphone systems, and other future incumbent users [11], [14], [15]. White space devices (WSDs) should either sense the presence of primary signals or make use of a geolocation database to determine which spectrum is unused in the vicinity [5]. Current operational mechanism to discover available TV channels is using the geolocation database [16]. This is primarily because spectrum sensing is expensive in cost, energy consumption, and hardware complexity. In contrast, the geolocation approach does not require complex hardware and is easier to implement, where devices determine their locations and query a geolocation database that will return a list of available frequency channels and their associated maximum transmit powers at current location. However, geolocation database suffers from its inherent inefficiency. The geolocation approach uses propagation modelling to determine the available spectra, and hence, is very conservative in the channels it returns for a given location. Moreover, it can only protect registered systems, but PMSE users operate mostly on an unlicensed basis. For the concern of the speed of database update, the use of real-time spectrum sensing in addition to geolocation is proposed to resolve the issue for the efficient use of white space and harmonious coexistence with dynamic incumbent systems [17], [18]. 
In [19] and [20], hybrid frameworks are proposed to incorporate the advantages of both geolocation database and spectrum sensing, in which different spectrum sensing modules are sequentially performed at each channel after its occupancy is initially determined by the geolocation database. However, for the received signal over a wide frequency range, sequentially scanning the channels one by one will introduce a long sensing period and thus may cause missed opportunities or interferences [21]. Directly acquiring the wideband signals to detect spectral opportunities is thus desirable to capture the instant spectrum changes [5]. To alleviate the high sampling rate, compressive sensing was introduced to implement wideband spectrum sensing [22]. In [23], a database assisted compressive wideband spectrum sensing algorithm is proposed to reduce the sensing costs by employing the information from geolocation database to construct a non-iteratively reweighted least square signal reconstruction. Specifically, the channel historical power information from geolocation database is utilised for the weight calculation to replace the iterative process of weights updating in the iteratively reweighted least square (IRLS) algorithm. However, the resolution and accuracy of channel power information from geolocation database would affect the reconstruction accuracy in such a non-iterative way. Moreover, if unregistered PU appears or dynamic change of the spectrum status occurs, the error in the prior information from geolocation database would severely degrade the recovery performance.

Motivated by the above challenges, a low-complexity hybrid scheme of sub-Nyquist wideband spectrum sensing with geolocation database is explored in this paper for the effective use of white spaces within the coexistence of dynamic incumbent systems, such as the wireless microphones that do not register in the database. Recent works show that the use of additional prior information on the support in compressive sensing has advantages in terms of number of required measurements and computational complexity during the reconstruction stage [24]. To relax the sensing sensitivity required on the sensor node, prior TV channel occupancy status from geolocation database is utilised in the sensing process. With the assists from geolocation database, part of the complexity of local wideband sensing is transferred to the core network, thus decreasing the processing complexity and energy consumption required on the spectrum sensing.

To incorporate the channel status information from geolocation database, sub-Nyquist wideband spectrum sensing scheme based on multicoset sampling is exploited [25], in which a lowdimensional measurement matrix is computed to locate the occupied channels through recovering its support based on the jointly sparse nature of multiband signals. Two reconstruction algorithms, greedy algorithm and $\ell_{v}$-norm $(0<v<1)$ minimisation, are employed in the recovery process. Theoretical analyses and simulation results show that the proposed hybrid scheme speeds up the sensing process with enhanced detection performance and smaller sampling rate, while the use of spectrum sensing can track the changes of spectrum occupancy state in real-time. This is specially important when the prior information from geolocation is not perfectly reliable, so the instant channel occupancy state provided by spectrum

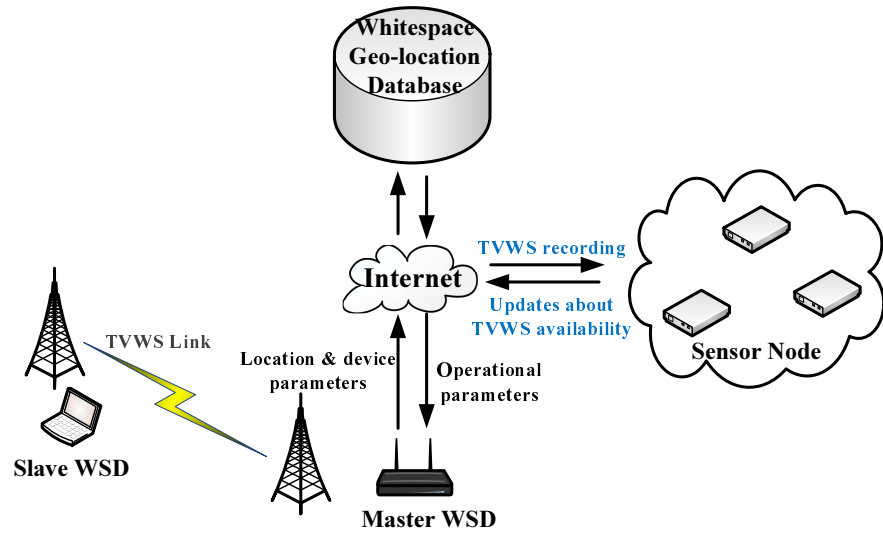

Fig. 1: System architecture of the proposed hybrid scheme.

sensing reduces the risks of WSDs interfering with dynamic incumbent users.

\section{System ARChitecture AND Problem FORMULATION}

\section{A. Signal Model}

Assume that the observed signal $x(t)$ is given by:

$$
x(t)=s(t)+n(t),
$$

where $s(t)$ is the primary signal and $n(t) \sim \mathcal{N}\left(0, \sigma_{n}^{2}\right)$ is the additive white Gaussian noise with zero mean and variance $\sigma_{n}^{2}$.

The received signal $x(t)$ is assumed to be a continuous-time wideband signal. Since the wideband spectrum is normally under-utilised in reality, $x(t)$ bears a sparse property in the frequency domain such that all (or most) of its energy is concentrated in one or more disjoint frequency bands and its spectral measure is small relative to the overall signal bandwidth.

Without loss of generality, the wideband spectrum to be monitored is evenly segmented into $M$ narrowbands, each of them with bandwidth $\mathcal{B}_{0}$. The channels are indexed from 0 to $M-1$. For TVWS spectrum regulation in UK, each channel is $8 \mathrm{MHz}$ and there are $M=40$ channels ranging from 470 to $790 \mathrm{MHz}$. Suppose there are up to $\kappa$ active channels occupied during the sensing period with $\mathcal{S}=\left[\mathcal{S}_{1}, \mathcal{S}_{2}, \ldots, \mathcal{S}_{\kappa}\right]$ denoting the set containing the indices of the occupied channels. The spectrum utilisation ratio is defined as $\alpha$, then $\kappa=\alpha \cdot M$. The task of wideband spectrum sensing is to find the presences and locations of the license transmissions or equivalently locating the active channel set $\mathcal{S}$.

\section{B. System Architecture}

Fig. 1 shows the block diagram of the proposed hybrid scheme. The aim of this framework is to enable the WSDs to use spectrum in the TV bands at a particular location and time on a shared basis subject to ensuring that there would be a low probability of harmful interference to other spectrum users in the band, such as digital TV broadcast signals, or dynamic incumbent systems, e.g., unregistered wireless microphones. WSDs' operation in TVWS will be controlled 
by the geolocation database, where WSDs measure their geographical location and query a geolocation database in order to get information on available frequencies at this location. The operational scenario with WSDs can be described as a communication between a master device and a slave device. Master WSD can establish a direct communication link with the geolocation database and request operational parameters for the transmission of any slave WSDs that are located within its coverage area.

For the efficient use of white spaces within the coexistence of dynamic incumbent systems, a joint scheme of geolocation database and spectrum sensing is explored in this paper. The rightmost side of Fig. 1 shows the diagram of the sensor network, which can be accessed and configured remotely. Spectrum sensing is implemented on the sensor nodes to scan the overall spectrum in real-time, and reports the dynamic changes of spectrum occupancy status to the geolocation database. Furthermore, in the context of spectrum sensing, some of the frequency bands are heavily used by the primary users such as local radio stations, local TV stations, etc., so the related information at the geolocation database will be stable due to TV broadcasting arrangement in the long run (e.g., years). Therefore, although the side-information from geolocation database is possibly with some errors due to the dynamic changes of the spectrum state, such information can be incorporated at the sensing terminals to reduce the sensing costs.

\section{Problem formulation}

Exploiting the sparse nature of the wideband spectrum due to the under-utilisation in practice, sub-Nyquist sampling is employed to implement wideband spectrum sensing, which acquires wideband signals using the sampling rate lower than the Nyquist rate and then detects spectral opportunities using these partial measurements [26]. To reduce the computation complexity, the channel status information from geolocation database, i.e., the locations of occupied channels, will be incorporated in the sensing framework. The purpose of this paper is then to show how this prior information can be efficiently used in the sub-Nyquist wideband spectrum sensing.

The compressive measurement acquisition at each sensor node can be expressed by the following analytical model:

$$
\boldsymbol{y}=\boldsymbol{\Phi} \boldsymbol{x}+\boldsymbol{\xi},
$$

where $\boldsymbol{\Phi} \in \mathbb{R}^{m \times N}$ is the sensing matrix to collect the compressive measurements $\boldsymbol{y}$ from the original signal $\boldsymbol{x}, m \in \mathbb{Z}$ (with $k<m<N$ ) refers to the dimension of $\boldsymbol{y}$, and $\boldsymbol{\xi}$ is the noise perturbation, whose magnitude is constrained by an upper bound $\delta$, i.e., $\|\boldsymbol{\xi}\|_{2}<\delta$.

Under certain assumptions, e.g., restricted isometry property (RIP) on $\Phi$ [27], robust signal reconstruction with respect to (2) could be achieved as

$$
\min _{\boldsymbol{x} \in \mathbb{R}^{N}}\|\boldsymbol{x}\|_{0} \quad \text { subject to }\|\boldsymbol{\Phi} \boldsymbol{x}-\boldsymbol{y}\|_{2}^{2} \leq \delta \text {. }
$$

Recent works show that additional prior information on the signal can be employed in the sparse recovery framework to improve the recovery performance [28]. For instance, if incomplete or complete prior information on the support of the sparse domain is available, the sparse recovery framework can be modified to seek a signal that explains the measurements and whose support contains the smallest number of new additions to the known support $T$ [24], so the sparsest solution is given by

$$
\min \left\|(\boldsymbol{x})_{T^{\mathrm{c}}}\right\|_{0} \text { subject to }\|\boldsymbol{\Phi} \boldsymbol{x}-\boldsymbol{y}\|_{2}^{2} \leq \delta .
$$

The minimization problem in (3) is aimed at finding the sparsest solution that explains the measurements. In (4), the problem is modified to minimise the number of nonzeros in other positions only, i.e., those that do not belong to the known support T. An important property of (4) is that a solution $\overline{\boldsymbol{x}}$ is not strictly constrained to be nonzero in the locations specified by $T$, since the corresponding values are determined from the measurement constraints and the minimisation of the objective function associated to the remaining positions [24]. Therefore, if there are some errors in the support set $T$, i.e., some positions in $T$ actually do not belong to the support, the minimisation problem in (4) can still reconstruct the actual signal, but more measurements may be required compared to the case when no errors are present in the known support $T$.

Suppose that the support of $\boldsymbol{x}$ is denoted as $S$, and $k:=|S|$. The size of the known part of the support $t:=|T|$, the size of unknown support $u:=|U|$, and $U_{e}:=T \backslash S$ is the error in the known part with the size $e:=\left|U_{e}\right|$, so that $k=t+$ $u-e$. The theoretical lower bound for exact reconstruction based on $l_{0}$-minimization can be expressed with the restricted orthogonality constant $\delta$ as [24]

$$
\delta_{t+2 u}<1
$$

which is much weaker than that of the original sparse recovery $\delta_{2 k}<1$ [27] as the restricted orthogonality constant $\delta$ is nondecreasing, and $k \gg u, k \gg e$. Sufficient condition for exact reconstruction in terms of $\delta$ measures the theoretical minimum number of measurements needed. Therefore incorporating the prior known part of the support can reduce the number of measurements, so that the related sampling rate will be reduced for the power-limited devices.

To improve the recovery performance with reduced processing requirements, the information from geolocation database is incorporated in the proposed spectrum sensing scheme. By harnessing the benefits of both approaches, the hybrid framework is a promising solution for the efficient use of the white space with coexistence of dynamic incumbent systems, including digital TV broadcast signals and unregistered wireless microphones. Since the geolocation database stores available frequency channel lists and their associated maximum transmit powers at current location and time, this paper aims to study how to incorporate this channel occupancy status information in the sub-Nyquist wideband spectrum sensing to reduce the reconstruction complexity in terms of the sampling rate and computational costs.

The notation used is as follows. The superscripts $(\cdot)^{T},(\cdot)^{H}$ and $(\cdot)^{\dagger}$ denote transpose, Hermitian transpose and pseudoinverse of a matrix $\left(\boldsymbol{X}^{\dagger}=\left(\boldsymbol{X}^{H} \boldsymbol{X}\right)^{-1} \boldsymbol{X}^{H}\right)$, respectively. $\boldsymbol{A}_{i, j}$ is the $i$-th entry of the matrix $\boldsymbol{A} . \boldsymbol{a}_{i}$ is the $i$-th column of the 


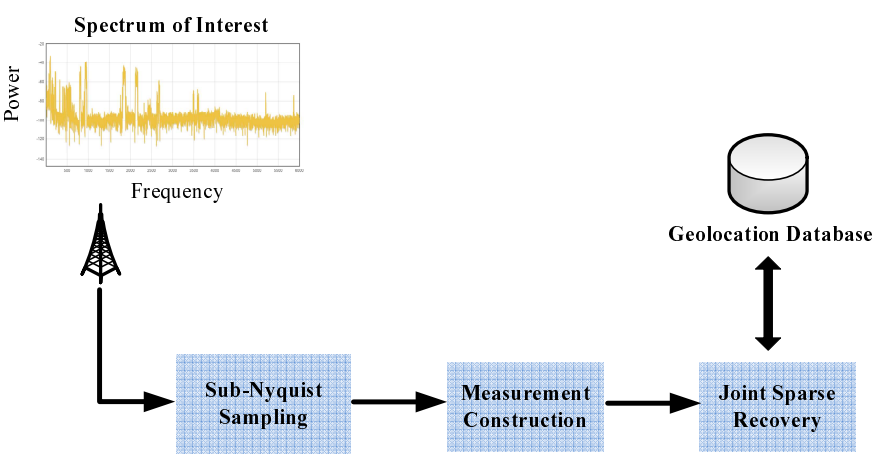

Fig. 2: Whole flowchart of the proposed scheme.

matrix $\boldsymbol{A} . \boldsymbol{A}[i]$ is the $i$-th row of the matrix $\boldsymbol{A} . \boldsymbol{A}_{\mathcal{T}}$ denotes the sub-matrix containing the columns of $\boldsymbol{A}$ with indices from $\mathcal{T}$. The notation, $[0, M-1]:=[0,1, \ldots, M-1] . \mathcal{T}^{c}$ denotes the complement of the set $\mathcal{T} . \mathcal{T}_{1} \backslash \mathcal{T}_{2}=\mathcal{T}_{1} \cap \mathcal{T}_{2}^{c}$ denotes the set difference. And $|\mathcal{T}|$ denotes the size of set $\mathcal{T}$.

\section{Sub-NyQuist WidEbAND SignAL SAMPLING}

To reduce the energy consumption in high frequency signal processing, compressive multicoset sampling is adopted in this paper to reduce the signal sampling and acquisition costs with ease of implementation. From the practical standpoint, multicoset sampling can be realized by a time-interleaved ADC [29], [30], in which only a subset of channels are used. The whole procedure of the proposed scheme can be formulated into a three-step framework, as shown in Fig. 2:

1) Each sensor implements a multicoset sampler that blindly samples the signal at a rate lower than the Nyquist rate.

2) A low-dimensional measurement matrix is constructed from sub-Nyquist samples.

3) Based on the jointly sparse nature of the multiband signals, the occupied channels are located through joint sparse recovery, where prior channel status information from geolocation database will be incorporated to relax the computation complexity.

In this section, we first briefly describe the sub-Nyquist signal sampling process based on multicoset sampling.

Given the number of channels $M$, multicoset sampling is executed by sampling the signal at the time instants $(m M+$ $\left.c_{i}\right) T$, where $i=1, \ldots, p, m \in \mathbb{Z}$, and $1 / T=f_{s}$ is the Nyquist sampling rate. The set $\mathcal{C}=\left\{c_{i}\right\}_{i=1}^{p}$ that consists of $p$ distinct integers randomly selected from $[0, M-1]$, is referred as a $(M, p)$ sampling pattern. Multicoset sampler can be realised by $p$ parallel cosets, each of them taking uniform samples at time instants $\left\{m M T+c_{i} T\right\}$ by a decimated sampling rate $\frac{1}{M T}=f_{s} / M$ with a time offset of $\left\{c_{i} T\right\}, i=1, \ldots, p$. The average sub-Nyquist sampling ratio equals to $\alpha=p / M$. The measurement sequence of the $i$-th coset is defined as

$$
x_{c_{i}}[n]=\left\{\begin{aligned}
x(n T), & n=m M+c_{i}, m \in \mathbb{Z} \\
0, & \text { otherwise. }
\end{aligned}\right.
$$

Applying Fourier transform to $x_{c_{i}}[n]$ gives the relationship between its spectrum $X_{c_{i}}\left(e^{j 2 \pi f T}\right)$ and the unknown Fourier Transform of $x(t)$ [31]:

$$
\begin{aligned}
X_{c_{i}}\left(e^{j 2 \pi f T}\right) & =\sum_{n=-\infty}^{+\infty} x_{c_{i}}[n] e^{-j 2 \pi f n T} \\
& =\frac{1}{M T} \sum_{m=0}^{M-1} \underbrace{X\left(f+\frac{m}{M T}\right)}_{X_{m}(f)} e^{j \frac{2 \pi}{M} c_{i} m} \\
& =\frac{1}{M T} \sum_{m=0}^{M-1} X_{m}(f) e^{j \frac{2 \pi}{M} c_{i} m} \quad \forall f \in\left[0, \mathcal{B}_{0}\right],
\end{aligned}
$$

for every $1 \leq i \leq p$, where $X_{m}(f)=X\left(f+\frac{m}{M T}\right)$ corresponds to the pieces of the original spectrum $X(f)$ in the $m$-th channel, which is shifted to the left by $\frac{m}{M T}$ units.

As $x(t)=s(t)+n(t)$, the corresponding Fourier transform can be expressed as $X(f)=S(f)+N(f)$. Define $S_{m}(f)=S\left(f+\frac{m}{M T}\right), m=0, \ldots, M-1$, and $\mathbf{S}(f)=$ $\left[S_{0}(f), S_{1}(f), \ldots, S_{M-1}(f)\right]^{T}$. Similarly we define $N_{m}(f)$ and $\mathbf{N}(f)$. We can rewrite (7) into the matrix form as

$$
\begin{aligned}
& \underbrace{\left[\begin{array}{c}
X_{c_{1}}\left(e^{j 2 \pi f T}\right) \\
X_{c_{2}}\left(e^{j 2 \pi f T}\right) \\
\vdots \\
X_{c_{p}}\left(e^{j 2 \pi f T}\right)
\end{array}\right]}_{\mathbf{Y}(f)} \\
& =\underbrace{\frac{1}{M T}\left[\begin{array}{cccc}
e^{\frac{j 2 \pi c_{1} 0}{M}} & e^{\frac{j 2 \pi c_{1} 1}{M}} & \cdots & e^{\frac{j 2 \pi c_{1}(M-1)}{M}} \\
e^{\frac{j 2 \pi c_{2} 0}{M}} & e^{\frac{j 2 \pi c_{2} 1}{M}} & \cdots & e^{\frac{j 2 \pi c_{2}(M-1)}{M}} \\
\vdots & \vdots & \vdots & \vdots \\
e^{\frac{j 2 \pi c_{p} 0}{M}} & e^{\frac{j 2 \pi c_{p} 1}{M}} & \cdots & e^{\frac{j 2 \pi c_{p}(M-1)}{M}}
\end{array}\right]}_{\mathbf{A}} \\
& \times \underbrace{\left[\begin{array}{c}
X_{0}(f) \\
X_{1}(f) \\
\vdots \\
X_{M-1}(f)
\end{array}\right]}_{\mathbf{X}(f)}=\mathbf{A}[\mathbf{S}(f)+\mathbf{N}(f)], \quad \forall f \in\left[0, \mathcal{B}_{0}\right],
\end{aligned}
$$

where $\mathbf{Y}(f)$ is a matrix whose $i$-th row is $X_{c_{i}}\left(e^{j 2 \pi f T}\right)$, $\mathbf{X}(f)=\left[X_{0}(f), X_{1}(f), \ldots, X_{M-1}(f)\right]^{T}$ is the unknown spectrum vectors of $x(t)$ in the $M$ channels, and $\mathbf{A} \in \mathbb{C}^{p \times M}$ is a matrix with $(i, j)$-th element given by $\mathbf{A}_{i, j}=\frac{1}{M T} e^{j \frac{2 \pi}{M} c_{i}(j-1)}$

Reconstruction of the unknown matrix $\boldsymbol{X}(f)$ in (8) is referred to as the multiple measurement vectors (MMV) problem, as $\boldsymbol{X}(f)$ is row-sparse, i.e., having nonzero entries in only a few rows. Since the parameter $M$ in the multicoset sampler is set based on the number of channels in the spectrum of interest, the positions of nonzero rows of in (8) is equivalent to the active channel index set $\mathcal{S}$. So here we can incorporate the channel status information from geolocation database in the recovery process, as it shows which part of channels are supposed to be occupied, in order to enhance the recovery performance with fewer measurements under subNyquist sampling. 


\section{JOINT SPARSE RECOVERY INCORPORATED WITH Geolocation Database}

The computation of the sparse solution in (8) is a MMV problem. Extension of the single measurement vector (SMV) to the MMV problem is considered in [32], where the objective is to minimise the number of rows containing nonzero entries while satisfying the measurement constrain in (8). The problem can be formulated as

$$
\min \left\|\mathcal{R}_{\ell_{q}}(\boldsymbol{X})\right\|_{0} \text { subject to } \boldsymbol{Y}=\boldsymbol{A} \boldsymbol{X},
$$

where $\mathcal{R}_{\ell_{q}}(\boldsymbol{X})$ is a vector in $\mathbb{R}^{M}$ whose $i$-th entry is the $\ell_{q}$ vector norm of the $i$-th row of $\boldsymbol{X}$, i.e.,

$$
\begin{aligned}
& \mathcal{R}_{\ell_{q}}(\boldsymbol{X})=\left[v_{1}, v_{2}, \ldots, v_{M}\right]^{T}, \\
& \text { where } v_{i}=\|\boldsymbol{X}[i]\|_{q}=\left(\sum_{j=1}^{N}\left|x_{i, j}\right|^{q}\right)^{1 / q} .
\end{aligned}
$$

As in the SMV problem, (9) is NP-hard but can be approximately solved by the following $\ell_{1}$ minimization problem:

$$
\min \left\|\mathcal{R}_{\ell_{q}}(\boldsymbol{X})\right\|_{1} \text { subject to } \boldsymbol{Y}=\boldsymbol{A} \boldsymbol{X} .
$$

Some existing convex relaxation and greedy algorithm for the sparse recovery problem have been proposed to extend to accommodate MMV problem [32], [33], which aims to solve $\boldsymbol{X}$ from the multiple-measurement vector $\boldsymbol{Y}$.

To enhance the recovery performance with fewer measurements under sub-Nyquist sampling, prior channel information from geolocation database is incorporated in the wideband spectrum sensing. Based on the white space channel information from the geolocation database, the sensor node can get a response with details of available channels in the vicinity. Assuming that $\mathcal{T} \subset[0, M-1]$ is the prior knowledge of the occupied channel indices from geolocation database, its relation to the actual occupied channel set $\mathcal{S}$ can be expressed as:

$$
\mathcal{S}=\mathcal{T} \cup \triangle \backslash \triangle_{e},
$$

where $\triangle:=\mathcal{S} \backslash \mathcal{T}$ is newly occupied channel set, and $\triangle_{e}:=$ $T \backslash S$ is the newly released channel indices, i.e., the occupied channel indices recorded at geolocation database but actually released as vacant at current time.

Motivated by the above challenges, two reconstuction algorithms, greedy algorithm and $\ell_{v}$ norm minimisation are modified in the MMV problem to incorporate the information from geolocation database in the recovery process. The aforementioned methods construct an estimate of the signal at each iteration, thereby being more intuitive to incorporate $\mathcal{T}$ in the recursion as an initial condition.

\section{A. Subspace-Augmented Joint Sparse Recovery with Prior Information}

To reduce computation costs in the compact sensor node, we firstly extend the greedy algorithm of simultaneous orthogonal matching pursuit (SOMP) [33] to this joint sparse problem because of its lower complexity compared with other $\ell_{1}$-minimization algorithms. To reduce the required number of measurements and the computational complexity, channel status information from geolocation database is incorporated in the reconstruction process to locate the occupied channels.
The channel occupancy status from geolocation database gives a priori information about some of the occupied channels that should be selected as they may be used or reserved by the registered systems. This way, local spectrum sensing is performed only on a limited number of potentially vacant TV channels.

Each multicoset sampler gets $p$ sample sequences $\boldsymbol{Y}(f) \in$ $\mathbb{C}^{p \times N}$. To further reduce the computation complexity of the recovery, we compute the covariance matrix of the sample sequences as [34]

$$
\boldsymbol{R}=\mathbb{E}\left[\boldsymbol{Y}(f) \boldsymbol{Y}^{H}(f)\right]=\boldsymbol{A}\left[\boldsymbol{R}_{s}+\sigma_{n}^{2} \boldsymbol{I}\right] \boldsymbol{A}^{H},
$$

where $\boldsymbol{R}_{s}=\mathbb{E}\left[\boldsymbol{S}(f) \boldsymbol{S}^{H}(f)\right]$ is the $M \times M$ primary signal correlation matrix. As there are up to $\kappa$ active channels occupied during the sensing period, $\boldsymbol{R}$ can be decomposed via the rank-revealing eigenvalue decomposition (RREVD) as [25]

$$
\boldsymbol{R}=\boldsymbol{U} \boldsymbol{\Lambda} \boldsymbol{U}^{H}=\boldsymbol{U}_{s} \boldsymbol{\Lambda}_{s} \boldsymbol{U}_{s}^{H}+\frac{\sigma_{n}^{2}}{M T^{2}} \boldsymbol{U}_{n} \boldsymbol{U}_{n}^{H},
$$

where $\boldsymbol{U}=\left[\boldsymbol{U}_{s}, \boldsymbol{U}_{n}\right], \boldsymbol{\Lambda}_{s}=\operatorname{diag}\left\{\lambda_{1}, \ldots, \lambda_{\kappa}\right\}$ contains the $\kappa$ non-increasing principal eigenvalues and $\boldsymbol{U}_{s}$ contains the corresponding eigenvectors, while $\boldsymbol{U}_{n}$ contains the corresponding eigenvectors associated with the smallest $p-\kappa$ eigenvalues $\frac{\sigma_{n}^{2}}{M T^{2}}$ related to the noise variance. As the noise term only perturbs the eigenvalues, the range of $\mathbf{R}$ spanned by $\mathbf{U}_{s}$, coincides with the signal subspace spanned by $\mathbf{A S}(f)$, and its orthogonal complement spanned by $\mathbf{U}_{n}$ is the noise subspace. Therefore, the $\kappa$ largest eigenvalues $\boldsymbol{\Lambda}_{s}$ and the corresponding eigenvectors $\mathbf{U}_{s}$ are chosen to construct the measurement matrix as $\chi_{s}=\mathbf{U}_{s} \sqrt{\boldsymbol{\Lambda}_{s}}$, and we can define the following linear system

$$
\chi_{s}=A \nu_{s}
$$

where the support of the sparsest solution to (15) converges to the original primary signal, i.e., $\operatorname{supp}\left(\boldsymbol{\nu}_{s}\right)=\operatorname{supp}(\mathbf{S}(f))$ [25]. As at most $\kappa$ active channels are assumed to be occupied by licensed users, $\boldsymbol{\nu}_{s}$ can be approximated to be jointly $\kappa$ sparse as it contains up to $\kappa$ significant rows. To reduce the computation complexity and improve the detection performance against noise permutation, SOMP is applied to the constructed low-dimensional measurement matrix $\chi_{s}$ in this paper, denoted as subspace-augmented SOMP (SA-SOMP). Compared with original sub-Nyquist samples $\boldsymbol{Y}(f) \in \mathbb{C}^{p \times N}$, using $\chi_{s} \in \mathbb{C}^{p \times \kappa}$ for recovery reduces the computation cost required on the sensor nodes. To avoid the threshold setting, exponential fitting test (EFT) is applied for the estimation of the support dimension $\hat{\kappa}$ [35].

In the recovery process of SA-SOMP, the column of $\boldsymbol{A}$ that is most strongly correlated with the remaining part of the signal is chosen at each iteration. Then we subtract off its contribution from the measurement matrix $\chi_{s}$ and iterate on the residual. Note that the indexes of the selected columns of $\boldsymbol{A}$ refers to the locations of nonzero rows of $\boldsymbol{\nu}_{s}$. As the parameter $M$ in the multicoset sampler is set based on the number of channels in the received signal, the support of $\boldsymbol{\nu}_{s}$ is equivalent to the active channel set $\mathcal{S}$. Therefore, based on the information from the geolocation database, the initialization of the support recovery is modified to subtract 


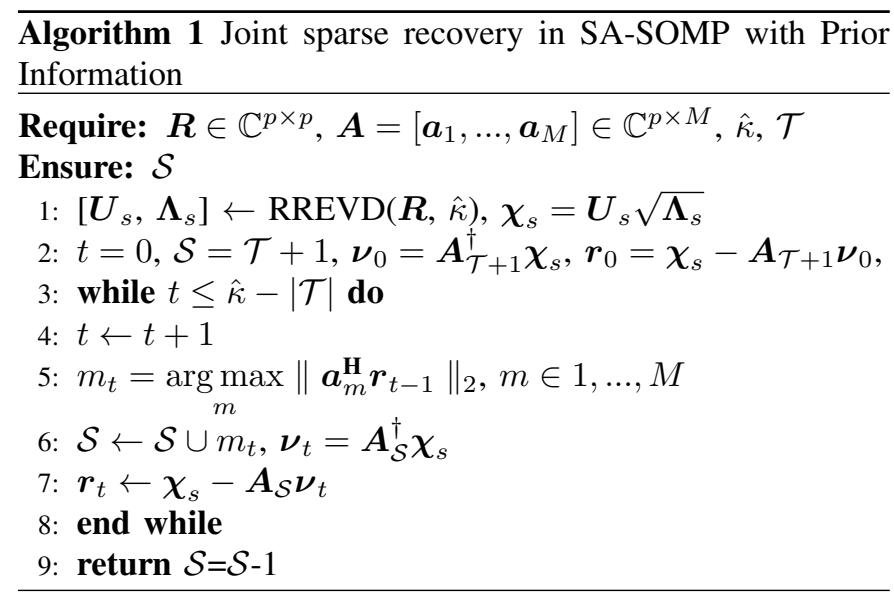

off the contributions of these corresponding columns in the measurement matrix as they may be used or reserved by the registered licence systems. Thus, before starting the iteration, the residual is initialized as

$$
\boldsymbol{r}_{0}=\chi_{s}-\boldsymbol{A}_{\mathcal{T}+1}\left(\boldsymbol{A}_{\mathcal{T}+1}^{\dagger} \chi_{s}\right),
$$

where $\mathcal{T} \subset[0, M-1]$ is added with 1 as the corresponding column indices of $\boldsymbol{A}$ start from 1. The entire procedure of the proposed joint sparse recovery based on SA-SOMP augmented by geolocation database is summarised in Algorithm 1. A sparsity-based halting criterion is used in the recovery process, i.e., $t \leq \hat{\kappa}-|\mathcal{T}|$. Therefore, with the TV channel information $\mathcal{T}$ from geolocation database, the number of iterations will be reduced, so that the joint approach could speed up the sensing process.

\section{B. Iterative Reweighted Support Detection with Prior Infor- mation}

Besides the $\ell_{1}$ norm, other functions of $\boldsymbol{X}(f)$ have also been proposed as objective functions. Among these algorithms, $\ell_{v}$ diversity measure $(0<v<1)$ has received great attention because of its improved performance as it provides a closer approximation to the $\ell_{0}$-norm minimisation. The objective function can be formulated as

$J^{v, q}(\boldsymbol{X})=\left\|\mathcal{R}_{\ell_{q}}(\boldsymbol{X})\right\|_{v}^{v}=\sum_{i=1}^{M}\left(\|\boldsymbol{X}[i]\|_{q}\right)^{v}, 0<v \leq 1, q \geq 1$.

The $\ell_{v}$ norm minimisation is nonconvex but can be solved through the transformation of the original problem into a sequence of convex problems using iterative reweighting [36]. In [37], a reweighted $\ell_{2}$ algorithm is proposed to extend FOCal Underdetermined System Solver (FOCUSS) to the MMV problem to minimise the above objective, with $q=2$, referred to as MFOCUSS. More specifically, the objective function in (9) is modified as a weighted least square problem in Lagrangian form:

$$
\min \|\boldsymbol{Y}-\boldsymbol{A} \boldsymbol{X}\|_{F}^{2}+\lambda \sum_{i=1}^{M} w_{i}\left(\|\boldsymbol{X}[\boldsymbol{i}]\|_{2}\right)^{2},
$$

where $\|.\|_{F}$ is the Frobenius norm, $w_{i}$ is the weighting parameter, and $\lambda$ is the Lagrange multiplier. The problem in (18) will be repeatedly solved by updating the weight $w_{i}$ at each iteration using the solution from previous iteration: at each iteration, $w_{i}$ will be set as

$$
w_{i}^{(t)}=\left(\left\|\boldsymbol{X}^{(t-1)}[i]\right\|_{2}\right)^{v-2} .
$$

where $w_{i}^{(t)}, i=1, \ldots, M$ is the value of the weighting vector to be used at the $t$-th iteration and $\boldsymbol{X}^{(t-1)}$ is the $(t-1)$-th iterate. After convergence, $\boldsymbol{X}^{(t-1)}$ will be sufficiently close to $\boldsymbol{X}^{(t)}$, so that $\sum_{i=1}^{M} w_{i}^{(t)}\left(\|\boldsymbol{X}[\boldsymbol{i}]\|_{2}\right)^{2}=$ $\sum_{i=1}^{M}\left(\left(\left\|\boldsymbol{X}^{(t-1)}[i]\right\|_{2}\right)^{v-2}\left(\|\boldsymbol{X}[\boldsymbol{i}]\|_{2}\right)^{2}\right.$ will be close to $\sum_{i=1}^{M}\left(\left(\left\|\boldsymbol{X}^{(t-1)}[i]\right\|_{2}\right)^{v}\right.$, which is the original $\ell_{v}$ norm problem in (17) with $q=2$.

The weighting parameter $\boldsymbol{w}^{(t)}$ are computed from the row norms of the solution obtained in the previous iteration, so the corresponding rows with smaller norm are likely to be deemphasised as they are irrelevant in fitting the data and vice versa. In (19), as $0<v<1$, the weights will be chosen inversely proportional to the $\ell_{2}$-norm of the rows. Since it gives a large weight to the small component, it will encourage a sparse solution in the minimisation problem of (18).

As shown in the proposed hybrid system model in the Fig. 1, the white space response from geolocation database records the channel occupancy status, while the sensing network will monitor the whole spectrum to find the dynamic changes. As the $i$-th row in $\boldsymbol{X}(f)$ corresponds to the piece of the original spectrum in the subchannel, the occupied channel information from geolocation database indicates the indices of the corresponding rows with large norm. Similar as (4), the objective function in (18) can therefore be changed as the $\ell_{v}$ minimisation over the remaining positions only, $i \notin \mathcal{T}$, i.e.,

$$
\min \|\boldsymbol{Y}-\boldsymbol{A} \boldsymbol{X}\|_{F}^{2}+\lambda \sum_{i \notin \mathcal{T}} w_{i}\left(\|\boldsymbol{X}[i]\|_{2}\right)^{2} .
$$

By defining

$$
w_{i}=0, \forall i \in \mathcal{T},
$$

the minimisation in (18) is transformed in the form of (20).

Here, in order to add the prior channel occupancy information from geolocation database, the weighing strategy in the joint sparse reconstruction is modified as

$$
w_{i}^{(t)}=\left\{\begin{array}{r}
\tau\left(\left\|\boldsymbol{X}^{(t-1)}[i]\right\|_{2}\right)^{v-2}, \quad i \in \mathcal{T} \\
\quad\left(\left\|\boldsymbol{X}^{(t-1)}[i]\right\|_{2}\right)^{v-2},
\end{array}\right.
$$

where $\tau$ is a specified small constant. For $\tau=0$, the first expression in (22) reduces to 0 as required by (21).

Given an initial guess of the signal $\boldsymbol{X}^{(0)}$ (e.g., the leastsquares solution), the iterative reweighting algorithm generates a sequence of iterations of as follows:

$$
\boldsymbol{X}^{(t+1)}:=\underset{\boldsymbol{X} \in \mathcal{C}^{M \times N}}{\arg \min }\|\boldsymbol{Y}-\boldsymbol{A} \boldsymbol{X}\|_{F}^{2}+\lambda \sum_{i \notin \mathcal{T}} w_{i}\left(\|\boldsymbol{X}[i]\|_{2}\right)^{2} .
$$

The solution to (23) at the $t$-th iteration can be expressed as

$$
\boldsymbol{X}^{(t+1)}=\boldsymbol{W}^{(t)} \boldsymbol{A}^{T}\left(\boldsymbol{A} \boldsymbol{W}^{(t)} \boldsymbol{A}^{T}+\lambda \boldsymbol{I}\right)^{-1} \boldsymbol{Y},
$$

where $\boldsymbol{W}^{(t)}=\operatorname{diag}\left\{\left[1 / w_{1}^{(t)}, \ldots, 1 / w_{M}^{(t)}\right]\right\}$. The initial weight is given by

$$
w_{i}^{(0)}=\left\{\begin{array}{lr}
\tau, & i \in \mathcal{T} \\
1, & \text { otherwise }
\end{array}\right.
$$




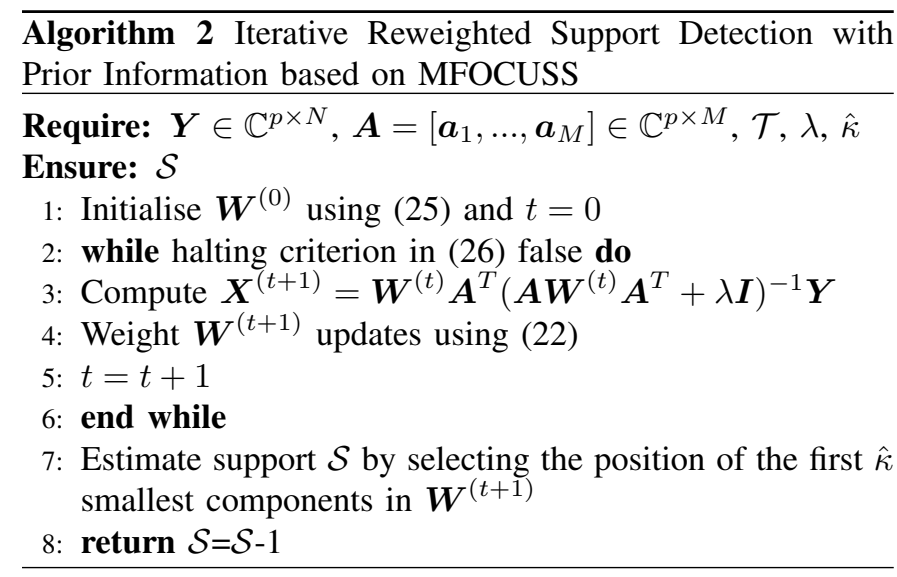

The algorithm is terminated once the convergence criterion has been satisfied, i.e.,

$$
\frac{\left\|\boldsymbol{X}^{(t+1)}-\boldsymbol{X}^{(t)}\right\|_{F}}{\left\|\boldsymbol{X}^{(t)}\right\|_{F}} \leq \delta,
$$

where $\delta$ is a user-selected parameter. Here, based on the sparsity guess of the support dimension $\hat{\kappa}$ from EFT [35], the estimated active channel set is determined by selecting the position of the first $\hat{\kappa}$ smallest components in the final weight $\boldsymbol{w}$. The entire procedure of the proposed joint sparse recovery based on MFOCUSS augmented by geolocation database is summarised in Algorithm 2.

\section{Numerical ANALYSis}

This section provides simulation results to evaluate the proposed wideband spectrum sensing scheme, in which the impact of different system parameters, such as the SNR, the sub-Nyquist sampling ratio, the spectrum occupancy ratio, and the detection performance with prior information from geolocation database, are investigated.

\section{A. Simulation Setup and Performance Measures}

Consider the received signal $x(t) \in \mathcal{F}=[0,320] \mathrm{MHz}$ containing $M=40$ channels of equal bandwidth $\mathcal{B}_{0}=8$ $\mathrm{MHz}$ and up to $\kappa \leq M$ active channels. The simulated signal is generated as

$$
x(t)=\sum_{i=1}^{\kappa} \sqrt{E_{i} \mathcal{B}_{0}} \operatorname{sinc}\left(\mathcal{B}_{0}\left(t-t_{i}\right)\right) e^{j 2 \pi f_{i} t}+n(t),
$$

where $\operatorname{sinc}(x)=\sin (\pi x) /(\pi x), E_{i}, t_{i}$, and $f_{i}$ define the energy, time offset, and carrier frequency respectively, on each active channel, and $n(t) \sim \mathcal{N}\left(0, \sigma_{n}^{2}\right)$ is the additive white Gaussian noise. The signal is observed on a time frame of $T=25.6 \mu \mathrm{s}$ starting at $t=0$, which corresponds to $T \cdot 320 \cdot 10^{6}=8192$ Nyquist rate samples. Fig. 3 depicts one example of the signal with $\kappa=4$ active channels, i.e., $\alpha=10 \%$. In this example, $E_{i}=\{1,1,1,1\}, t_{i}=\{4,8,16,20\} \mu \mathrm{s}$ and the spectrum support is centred at $f_{i}=\{36,92,156,212\}$ MHz. Thus the active channel set is $\mathcal{S}=[4,11,19,26]$.

To quantify the detection performance we compute the detection probability $P_{d}$, i.e., the fraction of occupied channels
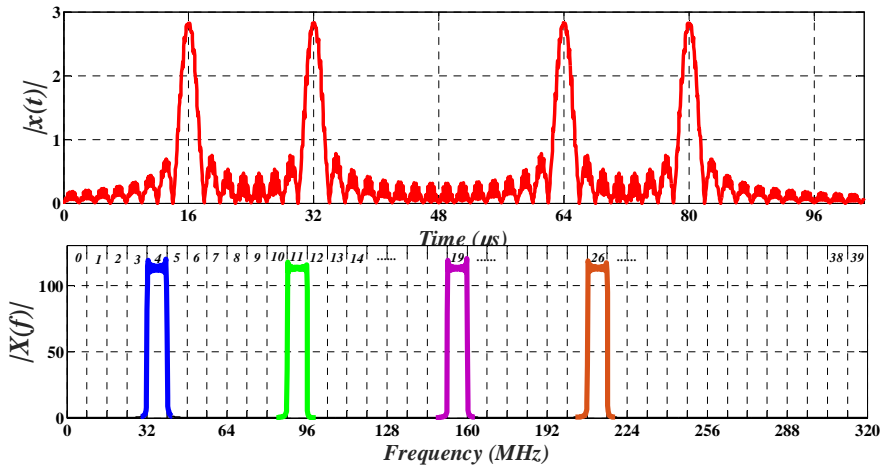

Fig. 3: Simulated signal illustration in time and frequency domains, with $M=40, \kappa=4$ and $\mathcal{S}=[4,11,19,26]$.

correctly being reported as occupied. The estimated active channel set $\hat{\mathcal{S}}$ is compared against the original signal support $\mathcal{S}$ to compute the detection probability under 2000 trials.

\section{B. Results and Analysis}

1) Spectrum Sensing Performance versus SNR and subNyquist Sampling Ratio: The joint sensing performance based on SA-SOMP and MFOCUSS is first evaluated in different conditions using the simulated signals with the number of occupied channels $\kappa=|\mathcal{S}|=0.2 M=8$. So the channel occupancy ratio $\alpha=20 \%$. The active channel set $\mathcal{S}$ are generated uniformly at random from $[0, M-1]$, among which the prior known part $\mathcal{T}$ from geolocation database of size $\tau$ are randomly chosen from the elements of $\mathcal{S}$. The size of $\mathcal{T}$, referred as $\tau$, is varied between 0 to $\kappa$. The case $\tau=0$ corresponds to the sensing only case without assists from geolocation database. The case $\tau=\kappa$ means current channel occupancy states from geolocation database are reliable and no change occurs on the spectrum at current time.

Firstly, the received SNR is set as $-5 \mathrm{~dB}$ and the number of cosets $p$ is varied in the multicoset sampler from 10 to 40 , corresponding to the sub-Nyquist sampling ratio $\Omega=p / M$ from $25 \%$ to $100 \%$. As shown in the Fig. 4 and Fig. 5, the detection performance generally increases with the number of cosets $p$, and also improves as the percentage of the known part $\tau$ increases. With geolocation database, the resulting curves of both schemes are shifted to the left, showing that smaller sub-Nyquist sampling ratios (smaller number of cosets in implementation) are required in the joint sensing scheme to achieve the same detection probability compared with the sensing only. When $p=15<2 \kappa(\Omega=37.5 \%)$, joint sensing scheme based on SA-SOMP achieve 0.93 above detection probability when $\tau \geq 0.5 \kappa$, while the sensing only has only 0.6 probability of correct detection. Compared with SA-SOMP, the joint sensing scheme based on MFOCUSS achieves better detection performance, as smaller number of cosets $p$ is needed for the same detection probability. To achieve the desired detection probability of 0.9 [38], sensing only based on SASOMP needs around $p=25$ cosets (sub-Nyquist sampling ratio $\Omega=62.5 \%$ ), and MFOCUSS needs around $p=20$ cosets (sub-Nyquist sampling ratio $\Omega=50 \%$ ), while the joint sensing schemes need only $p=15$ cosets. Moreover, joint sensing 


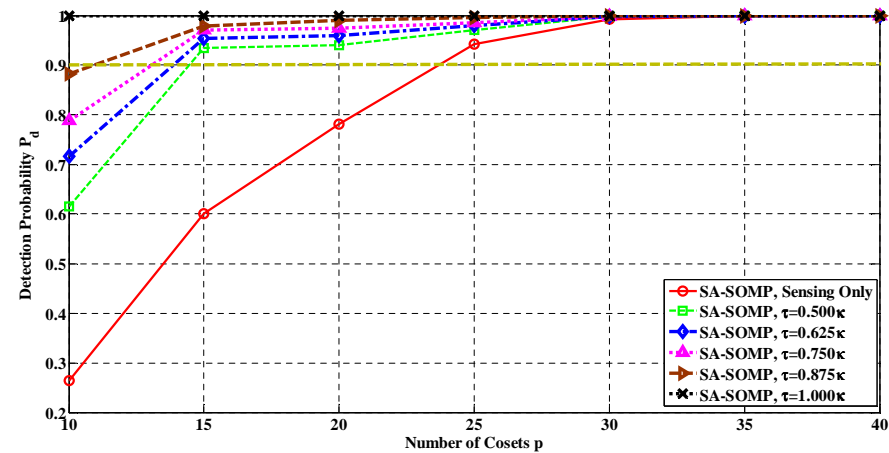

Fig. 4: Detection Probability $P_{d}$ vs. number of cosets $p$ with $\kappa=8$ and $\mathrm{SNR}=-5 \mathrm{~dB}$ under different number of occupied channels known from geolocation database based on SASOMP.

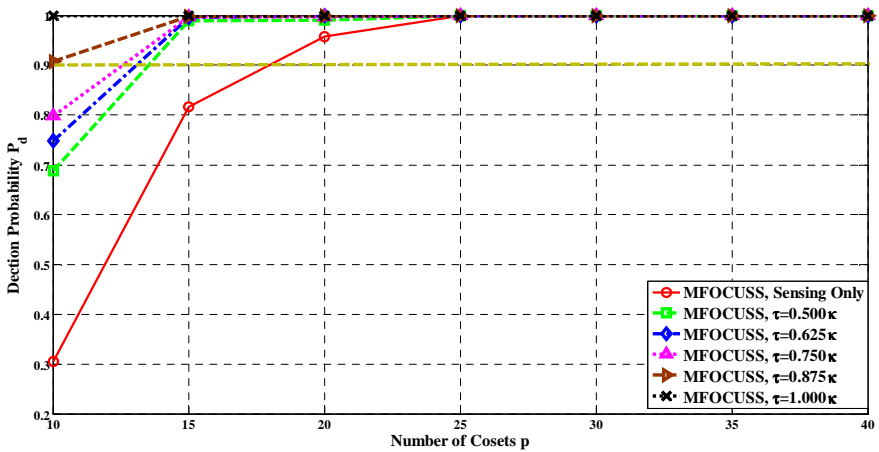

Fig. 5: Detection Probability $P_{d}$ vs. number of cosets $p$ with $\kappa=8$ and SNR $=-5 \mathrm{~dB}$ under different number of occupied channels known from geolocation database based on MFOCUSS.

can update the lack of channel occupancy information in the geolocation database, thus eventually reaching the desired detection probability $P_{d}$.

Then the detection performance is evaluated with varying received SNR from $-5 \mathrm{~dB}$ to $15 \mathrm{~dB}$. Assume geolocation database provides $50 \%$ above occupied channel information. Multicoset sampler with $p=15$ cosets is used to sample the received signals. As the results shown in the Fig. 6 and Fig. 7, the detection performance of the proposed joint sensing schemes are superior to that of the sensing only, especially more sensitive to the low levels of the SNR.

2) Average Iteration Number to Convergence: As shown in the Algorithm 1, a sparsity-based halting criterion is used in the recovery process based on SA-SOMP. Therefore, with the TV channel status information from geolocation database, the number of iterations will be reduced to $\hat{\kappa}-\tau$, so that the joint approach would speed up the sensing process.

Fig. 8 shows the average number of iterations in the proposed scheme based on MFOCUSS to achieve the convergence criterion in (26). By employing the prior information from geolocation database, the number of iterations in the joint sensing scheme is reduced for all values of $p$. As $\tau$ increases, the corresponding curve is shifted to the bottom. This shows that the prior information on the channel occupancy

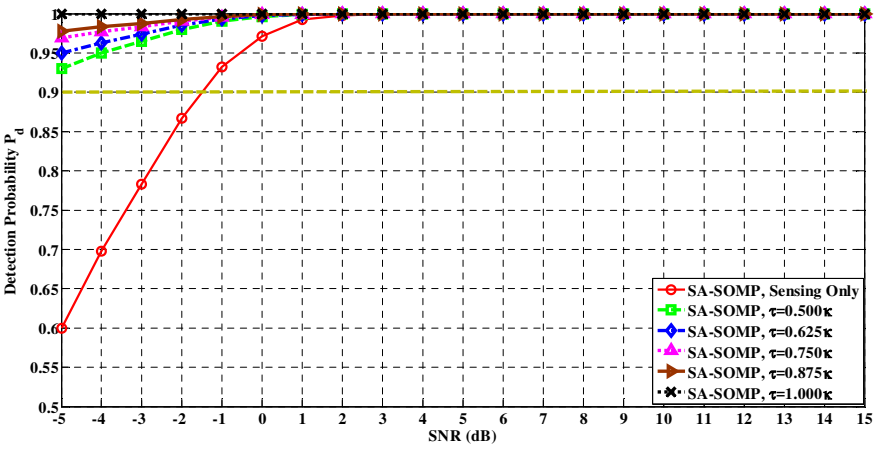

Fig. 6: Detection Probability $P_{d}$ vs. SNR with $\kappa=8$ and $p=15$ under different number of occupied channels known from geolocation database based on SA-SOMP.

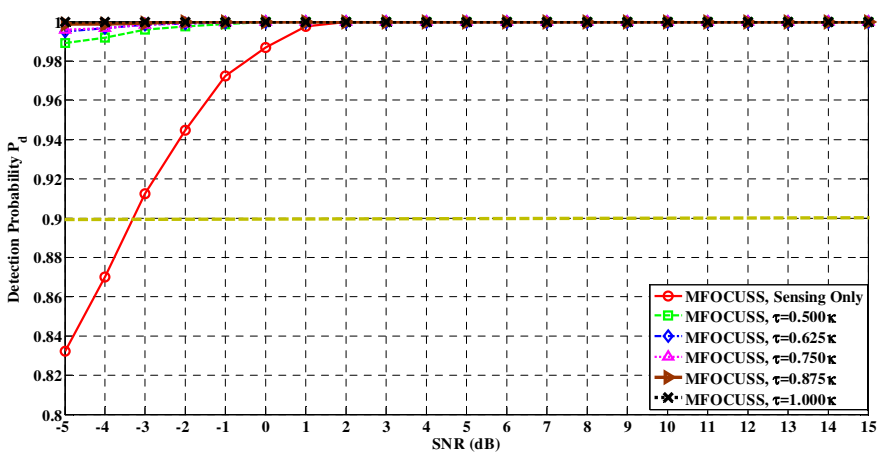

Fig. 7: Detection Probability $P_{d}$ vs. SNR with $\kappa=8$ and $p=15$ under different number of occupied channels known from geolocation database based on MFOCUSS.

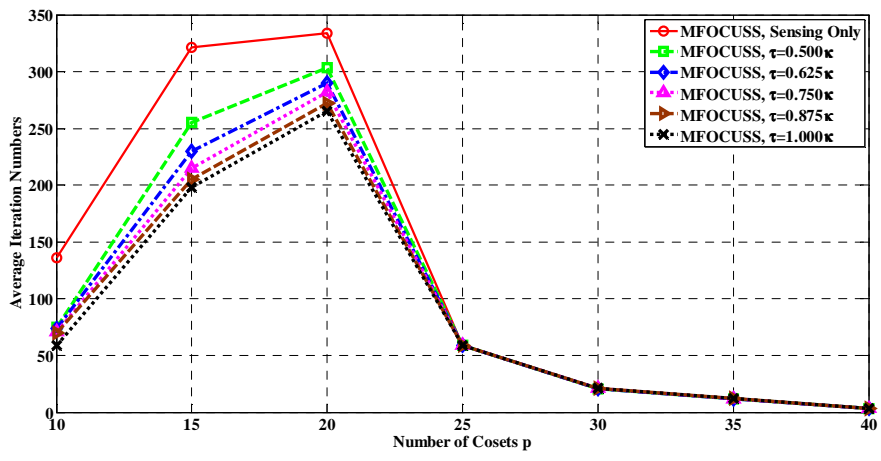

Fig. 8: Average number of iterations based on MFOCUSS to achieve convergence.

states from geolocation database is appropriately used by the proposed MFOCUSS method through the weighting scheme given by (22). The use of the prior information allows a reduction in the number of iterations, therefore reducing the total computation time to convergence.

Comparing the number of iterations in MFOCUSS with that in SA-SOMP, it shows that a better detection performance is achieved in MFOCUSS at the cost of more number of iterations required in the recovery process. In addition, at each iteration, the computation complexity of SA-SOMP is $O\left(p^{2} M\right)$, while it is $O(p M N)$ in the MFOCUSS. Therefore, SA-SOMP provides a more computation-efficient solution for 


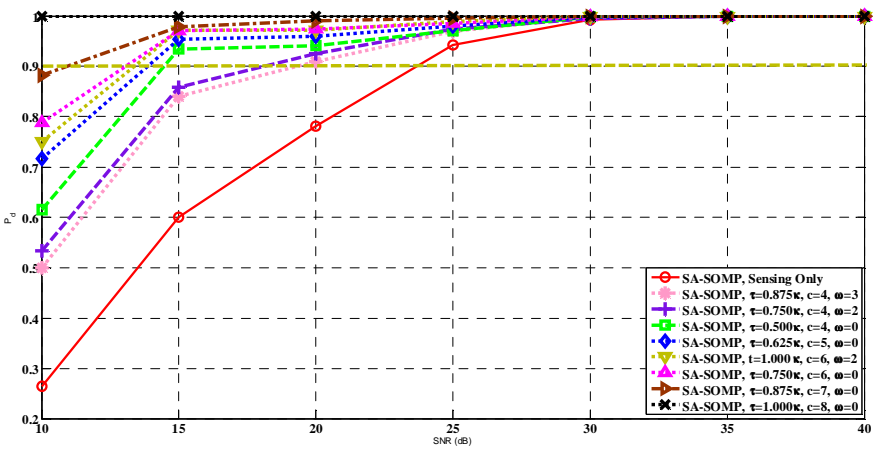

Fig. 9: Detection Probability $P_{d}$ vs. number of cosets $p$ with $\kappa=8$ and SNR $=-5 \mathrm{~dB}$ based on SA-SOMP under different number of occupied channels known from geolocation database with partially incorrect prior information.

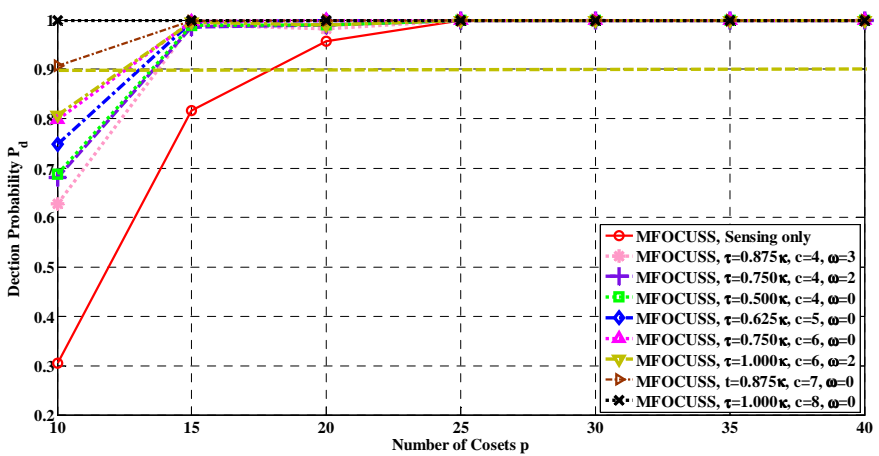

Fig. 10: Detection Probability $P_{d}$ vs. number of cosets $p$ with $\kappa=8$ and SNR $=-5 \mathrm{~dB}$ based on MFOCUSS under different number of occupied channels known from geolocation database with partially incorrect prior information.

the proposed joint sparse recovery.

3) Spectrum Sensing Performance with partially incorrect prior information in the geolocation database: Fig. 6 and Fig. 7 follows that the prior information from geolocation database is correct for all given channels. As stated in Section IV, it may be the case that the information from geolocation database is not fully reliable, so some of the channel occupancy states are changed at current time. In this situation, the joint sensing scheme can still recover the actual signals, but more measurements are required compared to the case when no errors are present in $\mathcal{T}$.

In Fig. 9 and Fig. 10, the cases in which $\mathcal{T}$ contains some incorrect prior information are simulated, which means that apart from the $c$ channels correctly belonging to the support, there are $\omega$ out of $\tau$ channels in $\mathcal{T}$ that do not belong to the current signal support. The simulation setting is same as that in Fig. 4 and Fig. 5, but with different combinations of $c$ and $\omega$ in $\mathcal{T}$. As shown in the Fig. 9 and Fig. 10, the joint sensing scheme can still reconstruct the underlying signals and shows an improvement in detection performance with respect to the case with no prior information. The joint sensing scheme based on MFOCUSS achieves better detection performance than that of the SA-SOMP, where the incorrect elements in $\mathcal{T}$ are removed from the minimisation problem.

\section{CONCLUSION}

In this paper, a low-complexity hybrid scheme of subNyquist wideband spectrum sensing with geolocation database is proposed for the effective use of white spaces within the coexistence of dynamic incumbent systems. The simulation results show that the prior channel status information from geolocation database leads to a reduction in the required subNyquist sampling ratio to achieve the desired detection performance. Moreover, the reduction in the number of iterations and computation time for convergence is also verified when prior information is added in the recovery process. If the prior information is not perfectly reliable, there is still an improvement in detection performance compared with spectrum sensing only. Therefore by harnessing the information from geolocation database, the proposed sensing scheme can update the instant channel occupancy state to reduce the risks of interference to the existing incumbent users. This makes it possible to apply the proposed joint sub-Nyquist scheme on compact devices to get accurate spectrum occupancy state with reduced processing requirements.

\section{ACKNOWLEDGMENT}

The authors would like to acknowledge the Engineering and Physical Sciences Research Council (EPSRC) in the UK for funding the work.

\section{REFERENCES}

[1] Y. Gao, Z. Qin, Z. Feng, Q. Zhang, O. Holland, and M. Dohler, "Scalable and Reliable IoT Enabled By Dynamic Spectrum Management for M2M in LTE-A," IEEE Internet of Things Journal, vol. 3, no. 6, pp. 11351145, Dec. 2016.

[2] R. N. Clarke, "Expanding Mobile Wireless Capacity: The Challenges presented by Technology and Economics," Telecommun. Policy, vol. 38, no. 8-9, pp. 693-708, Sep. 2014.

[3] J. Mitola and G. Maguire JR., "Cognitive Radio: Making Software Radios more Personal," IEEE Pers. Commun., vol. 6, no. 4, pp. 1318, Aug. 1999.

[4] A. B. Flores, R. E. Guerra, E. W. Knightly, P. Ecclesine, and S. Pandey, "IEEE 802.11af: A Standard for TV White Space Spectrum Sharing," IEEE Commun. Mag., vol. 51, no. 10, pp. 92-100, Oct. 2013.

[5] Office of Commun.. (Feb. 2015). Implementing TV White Spaces. [Online]. Available: http://stakeholders.ofcom.org.uk/binaries/ consultations/white-space-coexistence/statement/tvws-statement.pdf

[6] P. Kolodzy, "Spectrum Policy Task Force Report," Federal Commun. Commission, Nov. 2002, ET Docket No. 02-135.

[7] "Amendment of the Commission's Rules with Regard to Commercial Operations in the 3550-3650 MHz Band," Federal Commun. Commission, Sep. 2015, GN Docket No. 12-354.

[8] "In the Matter of Revision of Part 15 of the Commission's Rules to Permit Unlicensed National Information Infrastructure (U-NII) Devices in the $5 \mathrm{GHz}$ Band: Notice of Proposed Rulemaking," Federal Commun. Commission, Feb. 2013., ET Docket No. 13-49.

[9] O. Holland, S. Ping, Y. Gao, Z. Qin, A. Aijaz, J. Chareau, P. Chawdhry, and H. Kokkinen, "To White Space or not to White Space: That is the Trial within the Ofcom TV White Spaces Pilot," in Proc. IEEE Int. Conf. on Dynamic Spectrum Access Netw. (DYSPAN), Stockholm, Sweden, Sep. 2015, pp. 11-22.

[10] M. Fitch, M. Nekovee, S. Kawade, K. Briggs, and R. MacKenzie, "Wireless Service Provision in TV White Space with Cognitive Radio Technology: A Telecom Operator's Perspective and Experience," IEEE Commun. Mag., vol. 49, no. 3, pp. 64-73, Mar. 2011.

[11] Office of Commun. (Jul. 2009). Digital Dividend: Cognitive Access. [Online]. Available: http://stakeholders.ofcom.org.uk/binaries/ consultations/cognitive/statement/statement.pdf

[12] Y. Luo, L. Gao, and J. Huang, "Price and Inventory Competition in Oligopoly TV White Space Markets," IEEE J. Sel. Areas Commun., vol. 33, no. 5, pp. 1002-1013, May 2015. 
[13] G. Ding, J. Wang, Q. Wu, Y.-D. Yao, F. Song, and T. A. Tsiftsis, "Cellular-Base-Station Assisted Device-to-Device Communications in TV White Space," IEEE J. Sel. Areas Commun., vol. 34, no. 1, pp. 107-121, Jan. 2016.

[14] Y. Ma, Y. Gao, A. Cavallaro, C. G. Parini, W. Zhang, and Y.-C. Liang, "Sparsity Independent Sub-Nyquist Rate Wideband Spectrum Sensing on Real-time TV White Space," IEEE Trans. Veh. Technol., Apr. 2017 (to appear).

[15] Z. Qin, Y. Gao, M. Plumbley, and C. Parini, "Wideband Spectrum Sensing on Real-time Signals at Sub-Nyquist Sampling Rates in Single and Cooperative Multiple Nodes," IEEE Trans. Signal Process., vol. 64, no. 12 , pp. 3106-3117, Jun. 2016.

[16] Office of Communications. (Nov. 2010). Implementing Geolocation. [Online]. Available: https://www.ofcom.org.uk/_data/assets/pdf_file/ 0035/46889/statement.pdf

[17] B. Gao, S. Bhattarai, J.-M. Park, Y. Yang, M. Liu, K. Zeng, and Y. Dou, "Incentivizing spectrum sensing in database-driven dynamic spectrum sharing," in Proc. IEEE Int. conf. on Computer Commun. (INFOCOM), Apr. 2016, pp. 1-9.

[18] J. Wang, G. Ding, Q. Wu, L. Shen, and F. Song, "Spatialtemporal spectrum hole discovery: a hybrid spectrum sensing and geolocation database framework," Chinese Science Bulletin, vol. 59, no. 16, p. 18961902, Apr. 2014

[19] R. Dionisio, J. Ribeiro, P. Marques, and J. Rodriguez, "Combination of a Geolocation Database Access with Infrastructure Sensing in TV Bands," EURASIP J. Wireless Commun. Netw., vol. 2014, no. 1, pp. 1-14, Dec. 2014.

[20] N. Wang, Y. Gao, and B. Evans, "Database-Augmented Spectrum Sensing Algorithm for Cognitive Radio," in Proc. IEEE Int. Conf. on Commun. (ICC), London, UK, Jun. 2015, pp. 7468-7473.

[21] S. Yoon, L. E. Li, S. C. Liew, R. R. Choudhury, I. Rhee, and K. Tan, "QuickSense: Fast and Energy-efficient Channel Sensing for Dynamic Spectrum Access Networks," in Proc. IEEE Int. conf. on Computer Commun. (INFOCOM), Turin, Italy, Apr. 2013, pp. 2247-2255.

[22] Z. Tian and G. B. Giannakis, "Compressed Sensing for Wideband Cognitive Radios," in Proc. IEEE Int. conf. on Acoust., Speech and Signal Process. (ICASSP), vol. 4, Honolulu, HI, Apr. 2007, pp. 13571360.

[23] Z. Qin, Y. Gao, and C. G. Parini, "Data-Assisted Low Complexity Compressive Spectrum Sensing on Real-Time Signals Under SubNyquist Rate," IEEE Trans. Wireless Commun., vol. 15, no. 2, pp. 1174 1185, Feb. 2016.

[24] N. Vaswani and W. Lu, "Modified-CS: Modifying Compressive Sensing for Problems With Partially Known Support," IEEE Trans. Signal Process., vol. 58, no. 9, pp. 4595-4607, Sep. 2010.

[25] Y. Ma, Y. Gao, Y.-C. Liang, and S. Cui, "Reliable and Efficient SubNyquist Wideband Spectrum Sensing in Cooperative Cognitive Radio Networks," IEEE J. Sel. Areas Commun., vol. 34, no. 10, pp. 2750 2762, Oct. 2016.

[26] H. Sun, A. Nallanathan, C.-X. Wang, and Y. Chen, "Wideband Spectrum Sensing for Cognitive Radio Network: A Survey," IEEE Trans. Wireless Commun., vol. 20, no. 2, pp. 74-81, Apr. 2013.

[27] E. J. Candes and T. Tao, "Decoding by Linear Programming," IEEE Trans. Inf. Theory, vol. 51, no. 12, pp. 4203-4215, Dec. 2005.

[28] C. J. Miosso, R. von Borries, and J. H. Pierluissi, "Compressive Sensing With Prior Information: Requirements and Probabilities of Reconstruction in $l_{1}$-Minimization," IEEE Trans. Signal Process., vol. 61, no. 9, pp. 2150-2164, May 2013.

[29] S. M. Louwsma, A. J. M. van Tuijl, M. Vertregt, and B. Nauta, "A $1.35 \mathrm{GS} / \mathrm{s}, 10 \mathrm{~b}, 175 \mathrm{~mW}$ Time-Interleaved AD Converter in $0.13 \mu \mathrm{m}$ CMOS," IEEE J. of Solid-State Circuits, vol. 43, no. 4, pp. 778-786, Apr. 2008.

[30] K. Poulton, R. Neff, B. Setterberg, B. Wuppermann, T. Kopley, R. Jewett, J. Pernillo, C. Tan, and A. Montijo, "A 20 GS/s 8 b ADC with a 1 MB memory in $0.18 \mu \mathrm{m}$ CMOS," in Proc. IEEE Int. Solid-State Circuits Conf., vol. 1, Feb. 2003, pp. 318-496.

[31] P. Feng and Y. Bresler, "Spectrum-blind minimum-rate sampling and reconstruction of multiband signals," in Proc. IEEE Int. conf. on Acoust., Speech and Signal Process. (ICASSP), vol. 3, Atlanta, GA, May 1996, pp. 1688-1691.

[32] J. Chen and X. Huo, "Theoretical Results on Sparse Representations of Multiple-Measurement Vectors," IEEE Trans. Signal Process., vol. 54, no. 12, pp. 4634-4643, Dec. 2006.

[33] J. A. Tropp, A. C. Gilbert, and M. J. Strauss, "Simultaneous Sparse Approximation via Greedy Pursuit," in Proc. IEEE Int. conf. on Acoust., Speech and Signal Process. (ICASSP), vol. 5, Philadelphia, PA, Mar. 2005, pp. v/721-v/724.
[34] B. Nielson and D. R. Cox, Asymptotic Techniques for Use in Statistics, 1st ed. London; New York: Chapman and Hall/CRC, 1989.

[35] A. Quinlan, J.-P. Barbot, P. Larzabal, and M. Haardt, "Model Order Selection for Short Data: An Exponential Fitting Test (EFT)," EURASIP J. Adv. Signal Process., vol. 2007, Oct. 2006, Art. ID 71953.

[36] D. Wipf and S. Nagarajan, "Iterative Reweighted $\ell_{1}$ and $\ell_{2}$ Methods for Finding Sparse Solutions," IEEE J. Sel. Signal Process., vol. 4, no. 2 pp. 317-329, Apr. 2010.

[37] S. F. Cotter, B. D. Rao, K. Engan, and K. Kreutz-Delgado, "Sparse Solutions to Linear Inverse Problems with Multiple Measurement Vectors," IEEE Trans. Signal Process., vol. 53, no. 7, pp. 2477-2488, Jul. 2005.

[38] "IEEE Standard for Wireless Regional Area Networks Part 22: Cognitive Wireless RAN MAC and PHY specifications: Policies and procedures for operation in the TV Bands," IEEE Std. 802.22-2011, pp. 1-680, Jul. 2011. 